\title{
Eccrine Porocarcinoma: A Challenging Diagnostic and Therapeutic Tumoral Entity
}

\author{
Ha Mo Linh Le ${ }^{a} \quad$ Laurence Faugeras $^{a}$ Véronique De Moor ${ }^{b}$ \\ Caroline Fervaille $^{c}$ Thierry Vander Borght ${ }^{d}$ Fanny Collette ${ }^{a}$ \\ Lionel D'Hondt ${ }^{\mathrm{a}}$ \\ aDepartment of Oncology, CHU UCL Namur - Site Godinne, Yvoir, Belgium; bepartment \\ of General Surgery, CHU UCL Namur - Site Godinne, Yvoir, Belgium; 'Department of \\ Pathology, CHU UCL Namur - Site Godinne, Yvoir, Belgium; ${ }^{\mathrm{d} D e p a r t m e n t ~ o f ~ N u c l e a r ~}$ \\ Medicine, CHU UCL Namur - Site Godinne, Yvoir, Belgium
}

\section{Keywords}

Eccrine porocarcinoma $\cdot$ Sweat gland tumors - Management

\section{Abstract}

Eccrine porocarcinoma is a rare malignant cutaneous tumor with high rates of extracutaneous spread, and its diagnosis and management can be quite challenging. This is a case of an 82-year-old woman presenting with an asymptomatic and chronic pubic skin lesion for whom the work-up required many investigations and procedures to confirm the diagnosis of metastatic eccrine porocarcinoma. Indeed, the patient underwent a wide local excision of the skin lesion, imaging with an FDG-PET scan, a colonoscopy, and two inguinal node dissections. As illustrated in this case, surgery should always be considered to achieve disease remission. Other treatments such as chemotherapy and radiotherapy have also been reported in the literature without clear standard guidelines.

\section{Introduction}

Eccrine porocarcinoma (EPC) is a rare malignant cutaneous tumor with high rates of extracutaneous spread. It has first been described by Pinkus and Mehregan in 1963 [1]. EPC accounts for $0.005-0.01 \%$ of all malignant cutaneous tumors and arises from eccrine sweat

\section{Karger $\stackrel{\text { ! }}{=}$}


Fig. 1. A 2-cm ulcerated and erythematous skin lesion on the pubis.

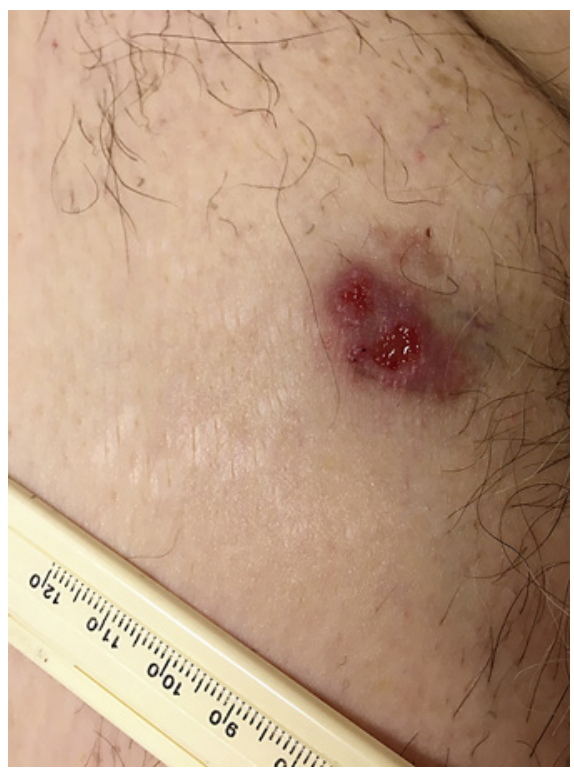

gland tumors [2]. Due to its uncommon onset, EPC can be misdiagnosed as other skin neoplasms, and optimal management is lacking.

Here we report a case of EPC with lymphovascular invasion managed in our institution. Multi-step treatment was required to obtain a complete remission.

\section{Case Report}

An 82-year-old Caucasian woman with no major past medical history was referred to our center after undergoing biopsies of longstanding pubic skin lesions. The patient was otherwise feeling well and had no symptoms. Physical examination revealed a 2-cm ulcerated lesion on her pubis (shown in Fig. 1). There were no palpable inguinal lymph nodes, and examination of the genital parts was normal.

The biopsy results from another medical center came back with a diagnosis of poorly differentiated squamous cell carcinoma on all the samples. The excision was incomplete, and therefore this patient was addressed for further management.

The patient's case was discussed at our multidisciplinary meeting, and wide local excision was decided. The histological report of the reexcision revealed a lesion with a depth of $4 \mathrm{~mm}$ in the dermis reaching the limit of the hypodermis, with tumoral cells infiltrating the dermis and harboring nuclear atypia and high mitotic activity. The lesion was ulcerative with a fibrinous leukocytic exudate. Vascular emboli were reported, and clear margins of $1 \mathrm{~cm}$ were achieved (shown in Fig. 2). Immunohistochemistry showed positive staining for carcinoembryonic antigen and cytokeratin (CK) 7 on the luminal ductal borders. p40 and CK5 and 6 were also positive on the whole cell population, and epithelial membrane antigen was heterogeneously positive. Those characteristics were consistent with the diagnosis of EPC. TNM classification was pT2.

Given the presence of vascular emboli, a staging CT scan of the thorax, abdomen, and pelvis was performed, which showed an image compatible with pelvic carcinomatosis. An additional FDG-PET scan was then requested, which displayed a hypermetabolic left inguinal lymph node and focal uptake in the right colon (shown in Fig. 3). Further investigations were

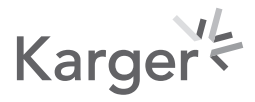




\section{Case Reports in Oncology}

\begin{tabular}{l|l}
\hline Case Rep Oncol 2021;14:700-705 \\
\hline DOI: 10.1159/000514984 & $\begin{array}{l}\text { @ 2021 The Author(s). Published by S. Karger AG, Basel } \\
\text { www.karger.com/cro }\end{array}$ \\
\hline
\end{tabular}

Le et al.: Eccrine Porocarcinoma: Challenging Management

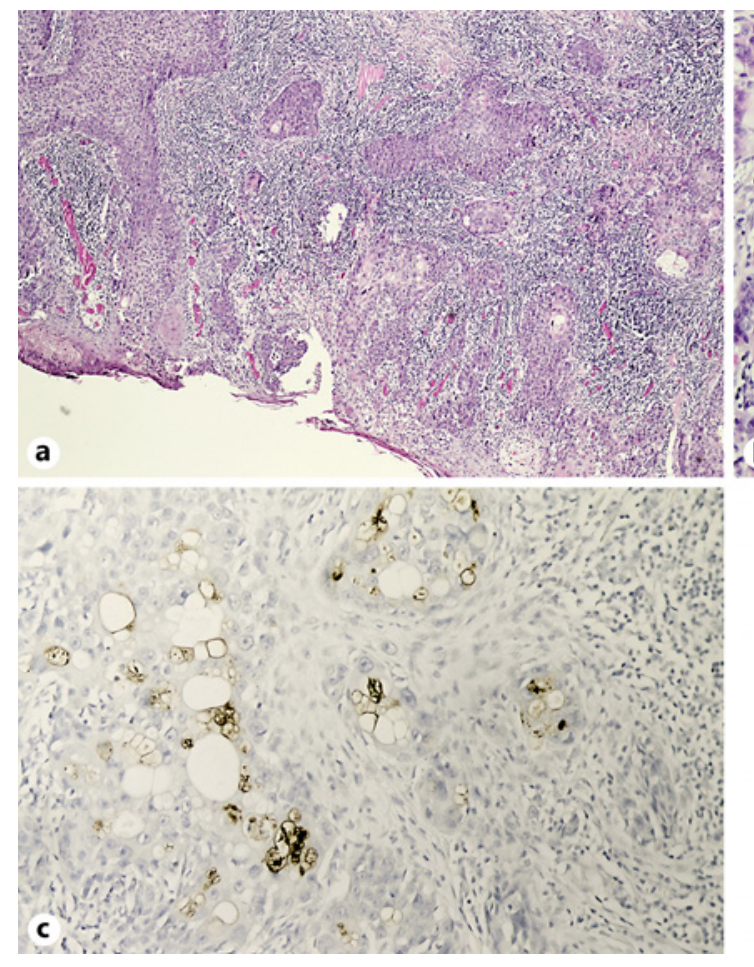

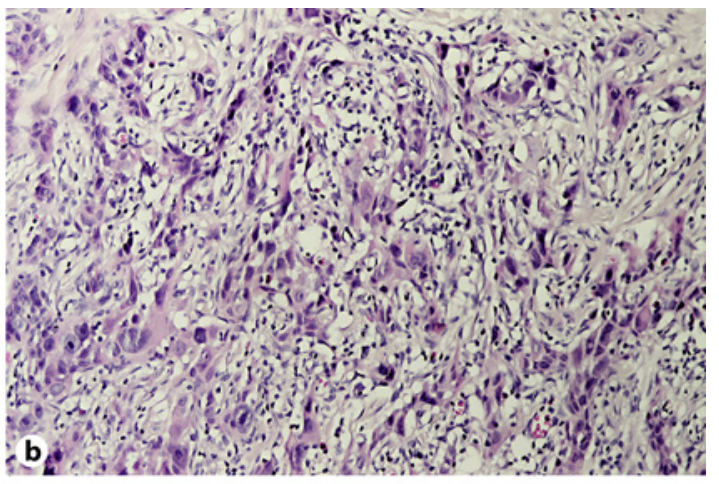

Fig. 2. a Hematoxylin and eosin-stained section of the skin $(\times 5)$. In situ and intradermal invasive components as well as dense dermal chronic inflammation are seen. $\mathbf{b}$ Hematoxylin and eosin-stained section $(\times 20)$ highlighting the infiltrative pattern and nuclear atypia. c Luminal ductal borders highlighted by immunohistochemistry for carcinoembryonic antigen.

conducted, which included a total colonoscopy and an inguinal left node biopsy. The ultrasound-guided node biopsy revealed distant infiltration of the porocarcinoma, whereas the endoscopy showed a large polyp which could not be endoscopically resected. Therefore, right colectomy combined with left inguinal and left iliac node dissection was performed, but the pathological findings showed no metastatic involvement. An additional ultrasound-guided resection was carried out to remove the targeted left hypermetabolic inguinal node, whose histology eventually revealed EPC and confirmed the disease's metastatic setting. The patient has been undergoing close follow-up for the last 4 months without any sign of relapse.

\section{Discussion}

EPC is an uncommon malignant adnexal cutaneous tumor deriving from terminal cells of the intraepithelial part of the eccrine duct called the acrosyringium. It occurs mainly in elderly patients, most frequently between the sixth and the eighth decade. It equally affects males and females and does not have any propensity for a specific ethnicity. There is currently no evidence suggestive of a hereditary factor. The etiology of EPC remains unknown, but risk factors such as radiation exposure and immunosuppression are correlated with EPC [3]. It arises de novo or develops from a benign preexistent lesion (poroma, nevus sebaceus, or actinic lesions). It is commonly found on the lower limbs and the head, but other sites have frequently been reported, such as the upper limbs, the trunk, and the abdomen. Head and neck locations seem to be associated with a lower incidence of lymph node involvement, whereas primary lesions on the genitalia and buttocks have a higher propensity for distant metastases [4].

Clinically, EPC appears as an asymptomatic erythematous or violaceous nodule or mass, although it can sometimes be ulcerative and painful [5]. It is often misdiagnosed as other skin lesions, particularly as squamous cell carcinoma. Other differential diagnoses include 


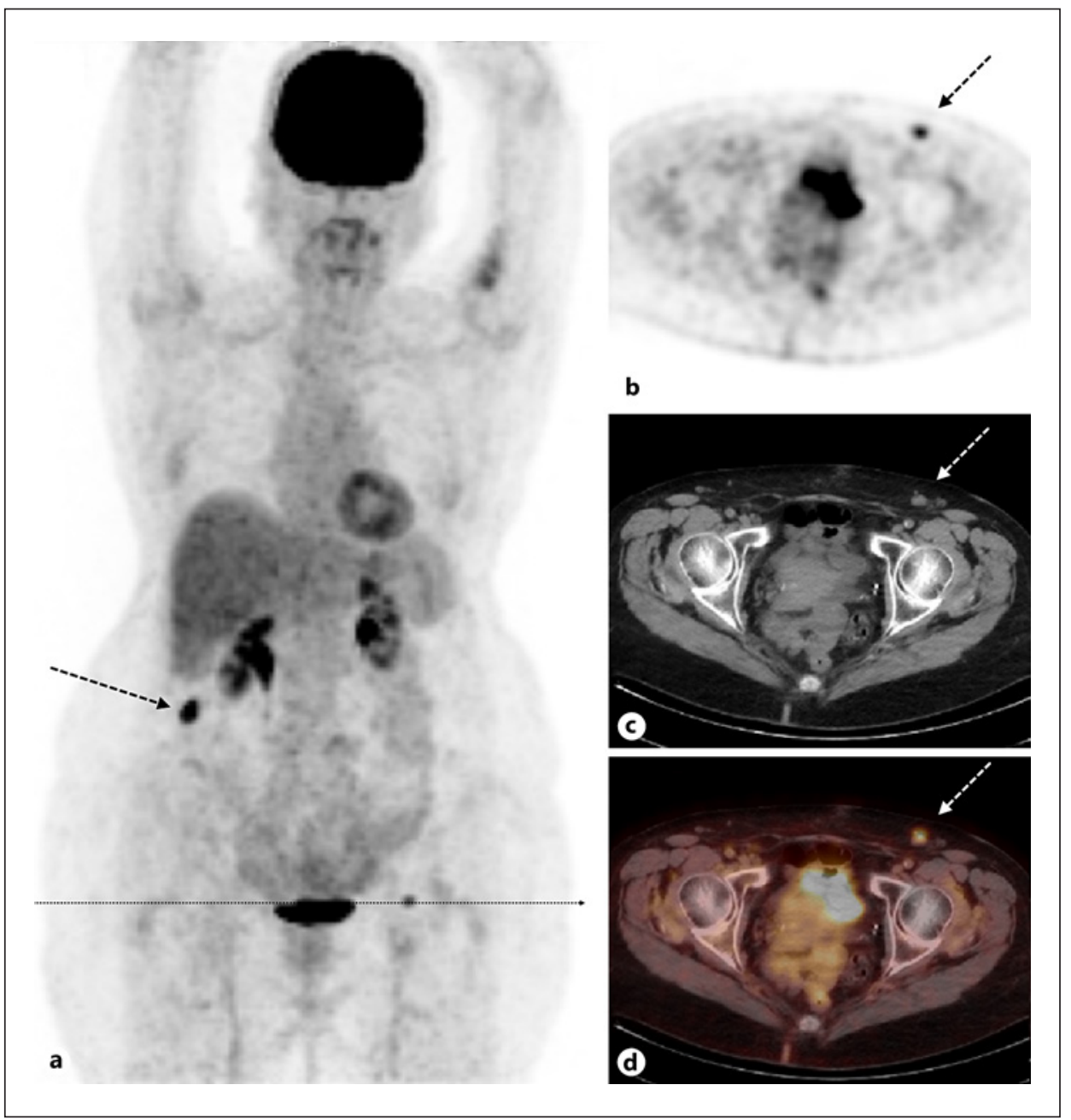

Fig. 3. In the left panel, the maximum-intensity projection image (a) shows foci of intense FDG uptake in the right colon (arrow) and in a left inguinal node through the level of which PET (b), noncontrast CT (c) and fused PET-CT (d) axial images are displayed. In the right panel, the left inguinal node is also highlighted by arrows on the three axial images.

pyogenic granuloma, amelanotic melanoma, seborrheic keratosis, Bowen's disease, fibroma, verruca vulgaris, or metastatic adenocarcinoma.

Given its rare occurrence, there is currently no guideline for its work-up and management. Most available data are based on case reports and small case series. Robson et al. [3] presented a review of the disease with the largest series of 69 patients, describing the clinicopathologic features of EPC. Histology is important to confirm the diagnosis of EPC. This tumor shows various histopathological features, but some common characteristics are found, including nuclear atypia, an infiltrative growth pattern, increased mitotic activity, and necrosis. The architecture presents ductal differentiation and nests of basaloid cells [3, 6]. EPC is classified into three histological variant groups according to the infiltrative pattern of the margins. There is the "infiltrative" variant, which is characterized by malignant clusters infiltrating the dermis and often the hypodermis, the "pushing" form with a clear lower dermal limit, and the "pagetoid" lesion with an intraepidermal spreading of neoplastic cells [3, 4]. A high mitotic index, lymphovascular invasion, and a tumor depth greater than $7 \mathrm{~mm}$ are histopathological features that 
have been associated with poor prognosis [3]. Immunohistochemistry is also a helpful tool, though there is no single marker for eccrine cells. These often show positive staining for keratin, epithelial membrane antigen, carcinoembryonic antigen, CK, p53, and p63 [3]. CD117 may be a useful marker in helping to distinguish EPC from squamous cell carcinoma [4]. The prognosis of EPC is not well established, due to its rarity and its varied clinical course. Fiveyear mortality rates reach $67 \%$ in case of positive regional lymph nodes [7].

Staging with radiology is useful for the evaluation of local and distant metastases, especially for tumors with high-risk features and poorly differentiated porocarcinoma. Varied modalities of diagnostic imaging are used, including whole-body CT scanning and PET-CT, but local imaging for lymph node assessment based on ultrasound, CT, or MRI has also been reported [4]. Sentinel lymph node biopsy remains controversial, but its use might be supported for high-risk EPC [8].

Treatment should always be considered, as it is an aggressive disease with a high potential for morbidity and mortality. Lymph node involvement is found in $20 \%$ of EPCs, and $10 \%$ have distant metastases at diagnosis [9]. The local recurrence rate is $20 \%$ in case of lymph node involvement [3]. Many treatment options have been reported, with no clear standard. Wide surgical excision is the mainstay of treatment, resulting in cure rates of $70-80 \%$ when the margins are clear. Some studies also reported good outcomes with Mohs micrographic surgery, and this technique could also be considered [4]. There is currently no evidence supporting adjuvant treatment, even in patients with poor prognostic factors [10]. Node dissection is recommended in the event of a positive sentinel node biopsy and clinical regional lymphadenopathy. Metastases occur mainly as cutaneous lesions in the primary area, but they can also develop in the lung, retroperitoneum, bone, liver, breast, bladder, peritoneum, or ovary [11]. Various chemotherapy regimens and radiation therapies have been used for the metastatic setting, but there is no standardized regimen. Treatment options are based on limited case series, and clinical responses have been achieved mostly with several combinations of chemotherapy [5, 8]. Piedbois et al. [12] achieved a durable complete remission using polychemotherapy with doxorubicin, mitomycin C, vincristine, and 5-fluorouracil (5-FU), alternating with cisplatin and bleomycin. In another case report, complete remission was seen in a patient with EPC of the leg after hyperthermic limb perfusion with 5-FU and melphalan [13]. A case of cutaneous metastatic EPC treated with topical 5-FU and intraarterial docetaxel has also been reported [11]. Plunkett et al. [10] showed some benefit using a single cytotoxic agent with docetaxel. More recently, responses have been reported with the use of epidermal growth factor receptor targeted therapy and pembrolizumab [14, 15].

\section{Conclusions}

EPC is a rare malignant skin disease for which tissue biopsy is essential for a diagnosis. This clinical case illustrates the fact that EPC is a challenging diagnosis, and that clinical features as well as histological characteristics are key to differentiate this disease from other skin tumors. Early diagnosis and treatment are essential to decrease mortality, as EPC has a high rate of recurrence and metastasis. As in our case, multistep treatment is sometimes required to obtain complete remission.

\section{Statement of Ethics}

The authors certify that written informed consent was obtained from the patient to publish this case and the images. 


\section{Conflict of Interest Statement}

The authors have no conflicts of interest to declare.

\section{Funding Sources}

There was no funding involved for this manuscript.

\section{Author Contributions}

H.M.L. Le reviewed the literature and wrote the Abstract, Introduction, Case Report, and Discussion sections. L. D'Hondt supervised the work. T. Vander Borght and C. Fervaille contributed to the figures' legends. L. D'Hondt, L. Faugeras, V. De Moor, T. Vander Borght, C. Fervaille, and F. Collette revised the paper and gave their final approval.

\section{References}

1 Pinkus H, Mehregan AH. Epidermotropic eccrine carcinoma. Arch Dermatol. 1963;88:597-606.

2 Wick MR, Goellner JR, Wolfe JT 3rd, Su WP. Adnexal carcinomas of the skin. I. Eccrine carcinomas. Cancer. 1985;56(5):1147-62.

3 Robson A, Greene J, Ansari N, Kim B, Seed PT, McKee PH, et al. Eccrine porocarcinoma (malignant eccrine poroma): a clinicopathologic study of 69 cases. Am J Surg Pathol. 2001;25(6):710-20.

4 Nazemi A, Higgins S, Swift R, In G, Miller K, Wysong A. Eccrine porocarcinoma: new insights and a systematic review of the literature. Dermatol Surg. 2018;44(10):1247-61.

5 Salih AM, Kakamad FH, Baba HO, Salih RQ, Hawbash MR, Mohammed SH, et al. Porocarcinoma; presentation and management, a meta-analysis of 453 cases. Ann Med Surg (Lond). 2017;20:74-9.

6 Riera-Leal L, Guevara-Gutiérrez E, Barrientos-García JG, Madrigal-Kasem R, Briseño-Rodríguez G, TlacuiloParra A. Eccrine porocarcinoma: epidemiologic and histopathologic characteristics. Int J Dermatol. 2015; 54(5):580-6.

7 Nguyen A, Nguyen AV. Eccrine porocarcinoma: a report of 2 cases and review of the literature. Cutis. 2014; 93(1):43-6.

8 De Iuliis F, Amoroso L, Taglieri L, Vendittozzi S, Blasi L, Salerno G, et al. Chemotherapy of rare skin adnexal tumors: a review of literature. Anticancer Res. 2014;34(10):5263-8.

9 Sawaya JL, Khachemoune A. Poroma: a review of eccrine, apocrine, and malignant forms. Int J Dermatol. 2014; 53(9):1053-61.

10 Plunkett TA, Hanby AM, Miles DW, Rubens RD. Metastatic eccrine porocarcinoma: response to docetaxel (Taxotere) chemotherapy. Ann Oncol. 2001;12(3):411-4.

11 De Bree E, Volalakis E, Tsetis D, Varthalitis Y, Panagiotidis J, Romanos J, et al. Treatment of advanced malignant eccrine poroma with locoregional chemotherapy. Br J Dermatol. 2005;152(5):1051-5.

12 Piedbois P, Breau JL, Morere JF, Israel L. Sweat gland carcinoma with bone and visceral metastases. Prolonged complete remission lasting 16 months as a result of chemotherapy. Cancer. 1987;60(2):170-2.

13 Briscoe KE, Grage T, Kennedy BJ. Sustained complete remission of metastatic sweat gland carcinoma following regional hyperthermic perfusion. JAMA. 1978;240(1):51-2.

14 Godillot C, Boulinguez S, Riffaud L, Sibaud V, Chira C, Tournier E, et al. Complete response of a metastatic porocarcinoma treated with paclitaxel, cetuximab and radiotherapy. Eur J Cancer. 2018;90:142-5.

15 Lee KA, Cioni M, Robson A, Bataille V. Metastatic porocarcinoma achieving complete radiological and clinical response with pembrolizumab. BMJ Case Rep. 2019;12(9):e228917.

\section{Karger'₹}

